\title{
Safety and Short-Term Oncological Outcomes of Thulium Fiber Laser En Bloc Resection of Non-Muscle-Invasive Bladder Cancer: A Prospective Non-Randomized Phase II Trial
}

Dmitry Enikeev ${ }^{\mathrm{a}, *}$, Mark Taratkin $^{\mathrm{a}}$, Vitaly Margulis ${ }^{\mathrm{b}}$, Nikolay Sorokin $^{\mathrm{a}}$, Lubov Severgina $^{\mathrm{c}}$, Nina Paramonova ${ }^{\mathrm{d}}$, Dmitry Kislyakova ${ }^{\mathrm{a}}$, Vasiliy Kozlov ${ }^{\mathrm{e}}$, Leonid Rapoport ${ }^{\mathrm{a}}$, Shahrokh F. Shariat ${ }^{\mathrm{a}, \mathrm{b}, \mathrm{f}, \mathrm{g}, \mathrm{h}}$ and Petr Glybochko ${ }^{\mathrm{a}}$

${ }^{a}$ Institute for Urology and Reproductive Health, Sechenov University, Moscow, Russia

${ }^{\mathrm{b}}$ Department of Urology, UT Southwestern Medical Center, Dallas, TX, USA

${ }^{\mathrm{c}}$ Department of Pathology, Sechenov University, Moscow, Russia

${ }^{\mathrm{d}}$ Centralized Pathology Department, Sechenov University, Moscow, Russia

${ }^{\mathrm{e}}$ Department of Public Health and Healthcare Organization, Sechenov University, Moscow, Russia

${ }^{\mathrm{f}}$ Department of Urology and Comprehensive Cancer Center, Medical University of Vienna, Vienna, Austria ${ }^{\mathrm{g}}$ Weill Medical College of Cornell University, New York, NY, USA

${ }^{\mathrm{h}}$ Department of Urology, Second Faculty of Medicine, Charles University, Prague, Czech Republic

Received 4 January 2020

Accepted 11 May 2020

Pre-press 6 June 2020

Published 11 June 2020

\begin{abstract}
.
BACKGROUND: Ongoing efforts aim at overcoming the challenges of conventional transurethral resection of bladder tumor (TURBT) such as the high recurrence rate, difficulty of pathologic interpretation and complications including wall injury. OBJECTIVE: To prospectively assess the safety and efficacy of Thulium fiber en bloc resection of bladder tumor (Tm-fiber ERBT) compared to TURBT.

MATERIALS AND METHODS: The prospective non-randomized study included 129 patients with non-muscle-invasive bladder cancer (NMIBC) divided into two groups: 58 patients underwent conventional TURBT and $71-$ Tm-fiber ERBT with FiberLase U1 (NTO IRE-Polus, Russia). Relapse-free survival (RFS), detrusor presence and complication rates were assessed. For multivariable analysis we used the Pearson chi-squared Hosmer-Lemeshow goodness of fit test; to compare survival - Cox regression analysis; for operative data comparison - chi-square test with Fisher's correction; for survival analysis - the Kaplan-Meier method and logrank test.

RESULTS: RFS rates at 3 and 6 months were $84.5 \%$ and $67.2 \%$ for conventional TURBT versus $97.2 \%$ and $91.5 \%$ for Tmfiber ERBT ( $p=0.011$ and $p<0.001$, respectively). Detrusor muscle was present in $58.6 \%$ of cases treated with conventional TURBT vs $91.6 \%$ for the Tm-fiber ERBT group $(p<0.001)$. The obturator nerve reflex and bleeding were noted in $17.2 \%$
\end{abstract}

\footnotetext{
*Correspondence to: Dmitry Enikeev MD, PhD, Deputy Director for Research, Institute for Urology and Reproductive Health,
} 
and $10.3 \%$ of TURBT cases, respectively; and in none of cases treated with Tm-fiber ERBT. Limitations included the non-randomized nature and the small sample size.

CONCLUSIONS: Tm-fiber ERBT seems to be a safe and efficacious treatment option for NMIBC. Tm-fiber ERBT had fewer adverse events, was more likely to secure detrusor muscle in the specimen and resulted in better RFS rates than conventional TURBT. Based on these promising data, we have started a prospective randomized clinical trial comparing en bloc TURBT with conventional TURBT (ClinicalTrials.gov NCT03718754).

Keywords: Bladder cancer, transurethral resection, thulium fiber laser, en bloc resection

\section{INTRODUCTION}

Bladder cancer is a growing problem for patients, healthcare providers and the society in general. Worldwide, it is the 7th most common malignancy in men and the 11th in both sexes [1]. Up to $80 \%$ of all primary detected bladder cancers are nonmuscle-invasive (NMIBC): Ta, T1, CIS [2]. The cornerstone of the diagnosis, staging and treatment of these tumors is the transurethral electroresection of the bladder tumor (TURBT) [3, 4]. The conventional TURBT, however, removes the tumors in piecemeal, breaking with the principles of oncology and all too often resulting in suboptimal (absence of detrusor)/incomplete resection $[4,5]$.

En bloc resection of bladder tumor (ERBT) was first employed by Kawada T. et al. in 1997. The technique showed promising results in terms of safety and tumor staging [6]. Development of laser surgery and emergence of the holmium (Ho:YAG) and later thulium (Tm:YAG) lasers in the 1990s enabled laser ERBT with subsequent studies demonstrating high efficacy and safety of the new techniques [7]. Ho:YAG and Tm:YAG ERBT have distinct advantages over conventional TURBT such as the complete absence of the obturator nerve reflex and hence, lower risks of perforation or bleeding as well as higher quality specimens for pathologic examination due to lack of the cautery effect [7]. Recently, a new type of thulium laser has been developed. It has decreased penetration depth $(<0.15 \mathrm{~mm})$ which is two times less than that of Tm:YAG [8]. Thermal damage to the sample is less marked, and the quasi-continuous mode of firing allows for minimal carbonization compared to Tm:YAG. These aspects show potential superiority of Tm-fiber laser over conventional solid-state lasers. However, current data on Tm:YAG laser ERBT for NMIBC is limited and that on Tm-fiber laser ERBT is absent altogether. We hypothesized that Tm-fiber laser ERBT would be characterized by fewer adverse events and better recurrence-free survival compared to conventional TURBT. To address this, we performed a prospec- tive phase II non-randomized comparative trial on the safety and efficacy of Tm-fiber ERBT and conventional TURBT. The co-primary endpoints were the recurrence rates at 3 and 6 months. Secondary endpoints included detrusor muscle in the specimen, the obturator nerve reflex and postoperative bleeding.

\section{MATERIALS AND METHODS}

\section{Patient identification and data collection}

A consecutive prospective cohort of 133 patients who underwent surgery for primary Ta or T1 NMIBC between February 2015 and December 2017 were included. Exclusion criteria were pure carcinoma in situ or invasion into muscle as seen on CT, MRI or pathology. Patients with intraoperative conversion from ERBT to TURBT were also excluded from the analysis. There was no limit in tumor size or number of tumors. Patient allocation to conventional TURBT or Tm-fiber ERBT was not based on any selection criteria (random allocation). The study was approved by the Sechenov University Institutional Review Board (Sechenov-ERBT-16). Informed consent was obtained from all individual participants included in the study.

\section{Surgical setup}

All surgeries were done by two experienced surgeons (both surgeons participating in the study had performed at least 50 ERBTs and 100 TURBTs prior to the beginning of the trial). Both of them performed conventional TURBT and Tm-fiber ERBT with a 26 FR resectoscope (Richard Wolf or Karl Storz, Germany) with constant irrigation. For laser ERBT, we used FiberLase U1 (NTO IRE-Polus, Russia), a thulium fiber laser with maximum power of $120 \mathrm{~W}$ and wavelength of $1.94 \mu \mathrm{m}$ (settings: energy at $1.0 \mathrm{~J}$, power at $10 \mathrm{~W}$ ) and a working element with an inner sheath for laser fiber $(600 \mathrm{~nm})$. 


\section{Surgical technique}

After cystoscopy and determining tumor topography, a circumferential incision around the tumor was made in the visually intact bladder mucosa (approximately $10 \mathrm{~mm}$ from the edges of the tumor). It allowed for accurate morphological evaluation of the surgical margin. After that, the incision was continued deeper into the muscular layer. Having identified the layer, the surgeon resected the base of the tumor with the muscular layer using traction and incisions of the muscle fibers. Making incisions in the muscular layer requires precision, a full bladder and adequate guidance with visualization techniques. Traction may be employed with the help of the tip of the resectoscope or working element. After complete tumor resection, local hemostasis was performed (Fig. 1). If resecting posterior tumors proved challenging, the surgeon emptied
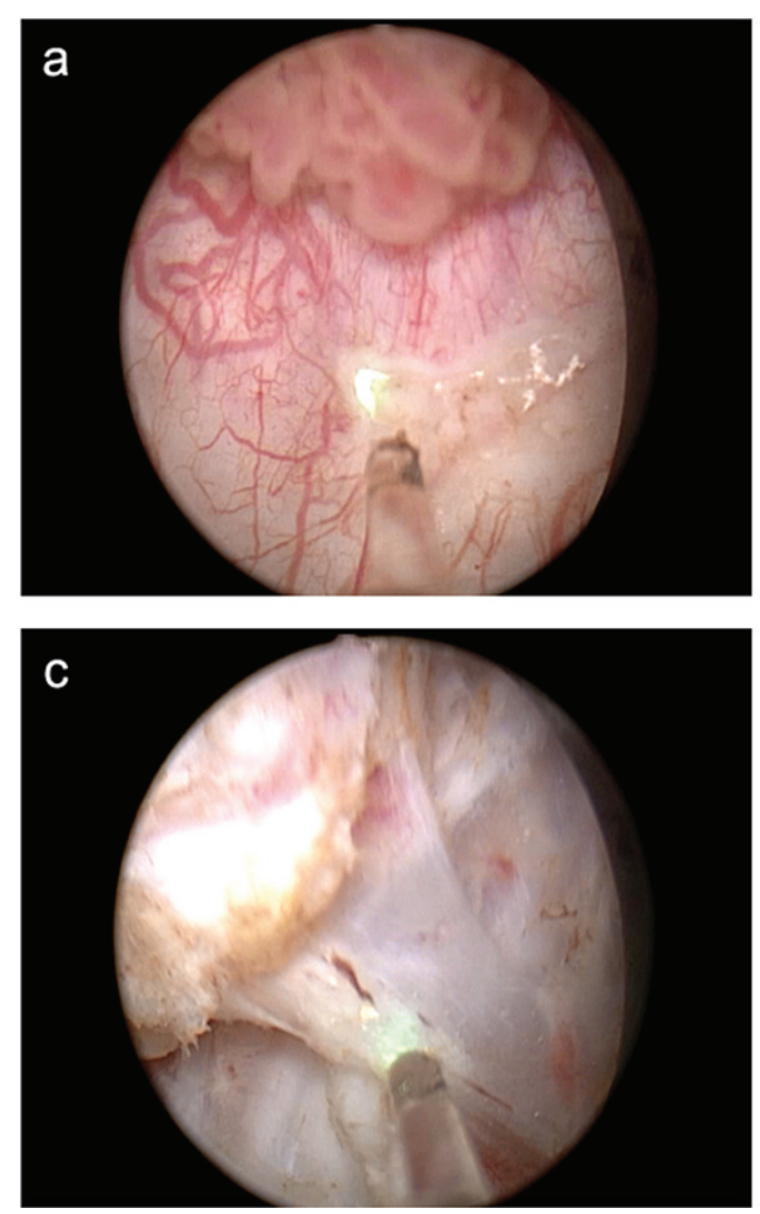

the bladder by half and put additional suprapubic pressure.

In cases of tumors larger than $3 \mathrm{~cm}$, we employed the two-step technique. The first step included resection of the larger exophytic area with the base of the lesion left intact. Then the tumor was removed with the help of morcellation. This part of the specimen was used for determining tumor morphology and for grading $(G)$. The second step was en bloc resection of the tumor base which was usually subsequently removed in one piece through the resectoscope. The technique follows the main principles of en bloc resection, which are obtaining a high-quality specimen for pathological examination and minimizing contact of the removed tumor with the intact bladder wall. The tumor was removed in one piece through the instrument with the help of the cold loop of the resectoscope, forceps, basket retrievers or endo-bag specimen retrieval system.
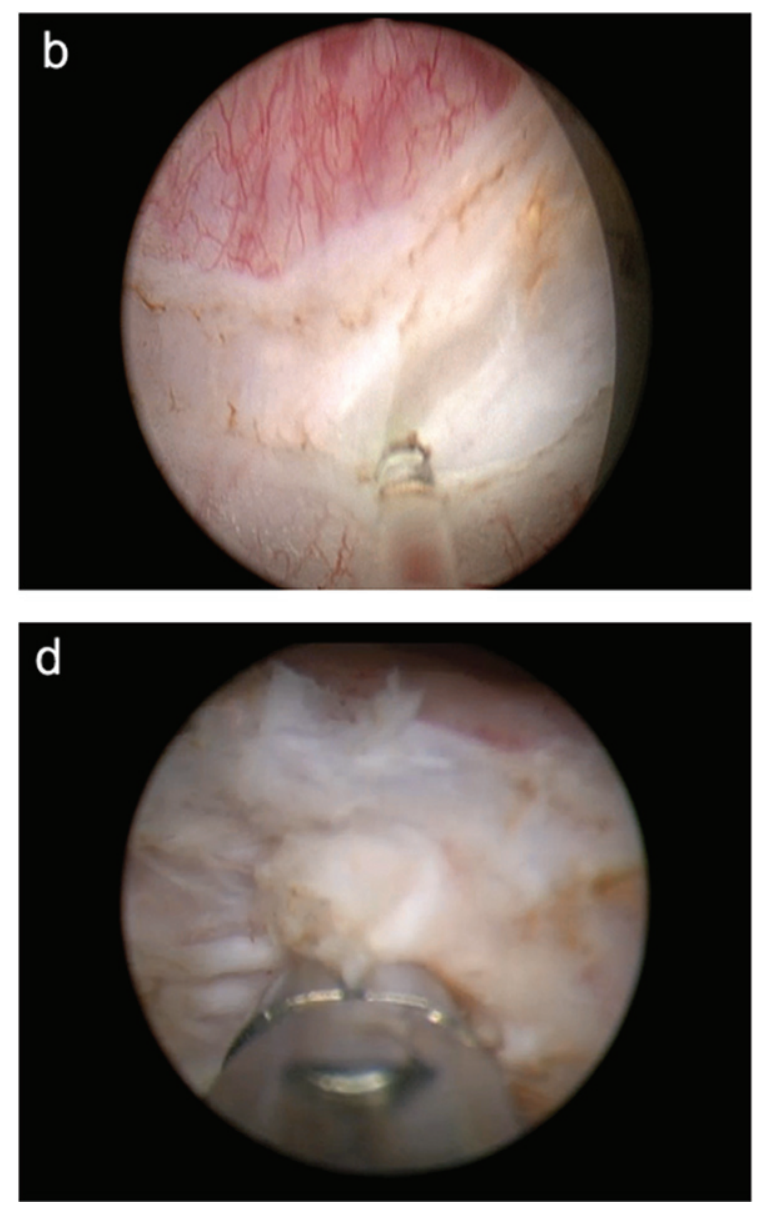

Fig. 1. Stages of en bloc resection: incision in the mucosa (a), incision in the muscular layer (b), resection of the tumor base from the surgical site (c), random biopsy from the surgical site (d). 
Conventional monopolar TURBT was performed with the following settings: $100-110 \mathrm{~W}$ for cutting and $60 \mathrm{~W}$ for coagulation. Step-by-step resection of tumor with muscularis propria was done, followed by additional resection of circumferential surgical margin. Tumors smaller than the diameter of the loop (about $5 \mathrm{~mm}$ ) were resected in one piece. Larger tumors were resected separately in fractions, which included the exophytic part of the tumor, the underlying bladder wall with the detrusor muscle and the edges of the resection area.

Upon completing TURBT or Tm-fiber ERBT and removing the resected fragment (circular or horizontal margin), biopsy was taken from a random area within the surgical site (vertical surgical margin). Follow up tests included cystoscopy at 3 and 6 months after surgery. Cystography was done postoperatively in patients with signs of bladder injury.

\section{Chemotherapy}

Immediate intravesical chemotherapy was given to all patients except in cases of perforation
( $40 \mathrm{mg}$ of mitomycin within the first two hours after surgery). Additional same-dose weekly adjuvant intravesical chemotherapy was given weekly in intermediate- and high-risk patients for eight weeks.

\section{Morphology assessment}

Tumor morphology and differentiation were evaluated according to the WHO classification (1973 and 2004). The specimen assessment was performed by the same uropathologist. Invasion beyond the basement membrane and surgical margins was also evaluated. The specimen obtained during en bloc resection included the following structures: tumor, basement membrane and a relatively large fragment of the underlying bladder wall with the submucosa and muscular layer. This allowed for accurate evaluation of invasion into the bladder wall, as well as perivascular and perineural invasion. Proper adherence to the surgery protocol also enabled assessment of circular and vertical surgical margins (Fig. 2).

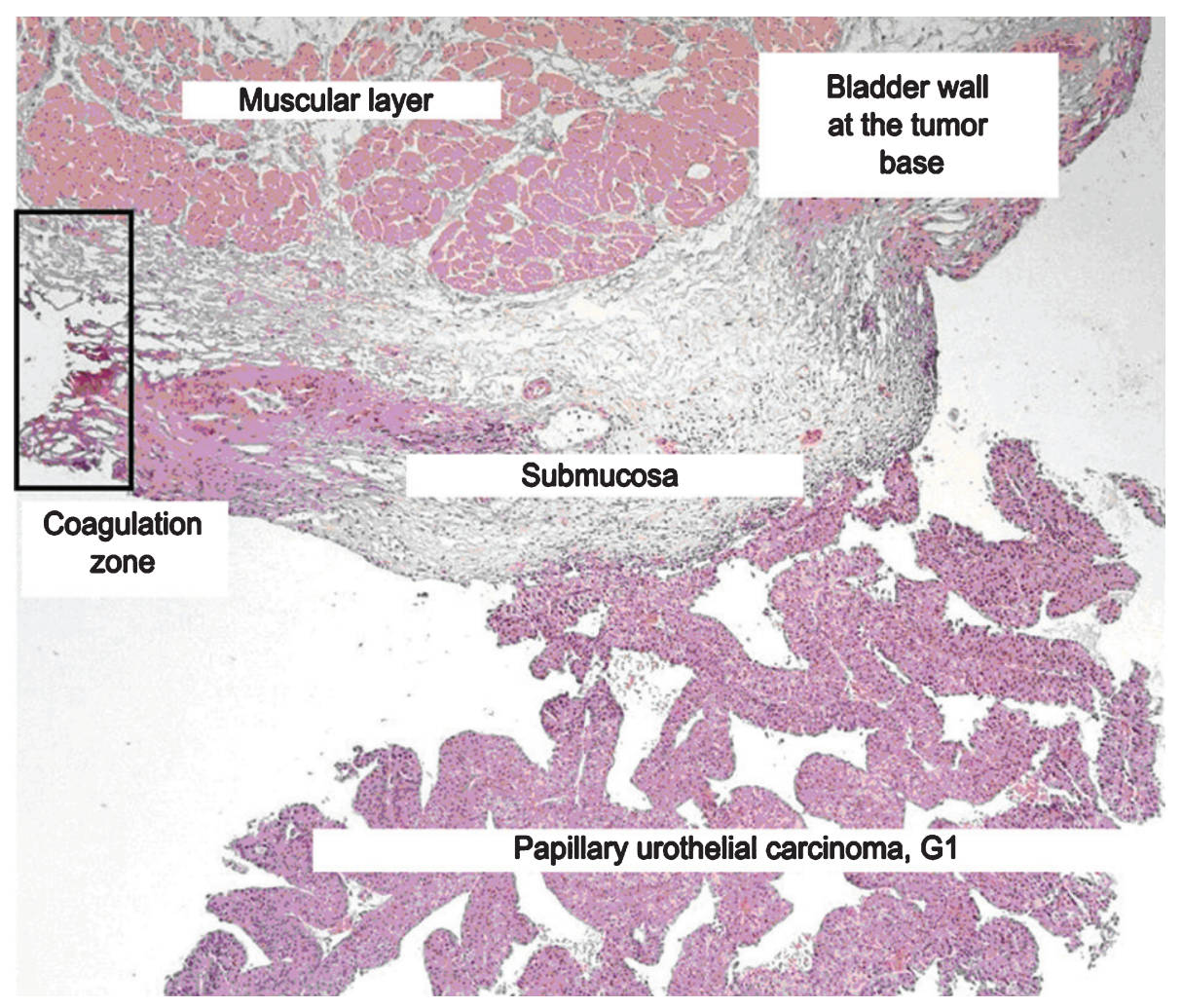

Fig. 2. Histology slide. 


\section{Primary endpoint}

Recurrence-free survival (RFS) rate assessed by means of outpatient cystoscopy at 3 and 6 months.

\section{Secondary endpoints}

Specimen quality (thermal damage, the presence of muscle fibers in specimens) and complications.

\section{Statistical analysis}

This was a phase II non-randomized prospective comparative clinical trial. There was no power calculation to assess power. Data are given as numbers and percentages, means and standard deviations, medians and interquartile ranges where appropriate. To predict RFS, multivariable analysis was performed that adjusted for the effects of standard clinicopathologic variables. The Pearson chi-squared Hosmer-Lemeshow goodness of fit test for logistic regression was used. To compare survival between groups, Cox regression analysis was used. Pre- and post-resection data were compared using the chisquare test with Fisher's correction when necessary. Confidence intervals of the means were calculated on the basis of Student's criterion with $p=0.05$. Survival analysis was conducted by Kaplan-Meier survival curves and logrank test (Mantel-Cox test). The time of recurrence was calculated from the date of surgery to the date of recurrence or last contact. All tests were two-sided, and $p$-values less than 0.05 were considered statistically significant. Statistical analyses were carried out with SPSS 23.0.

\section{RESULTS}

Patient data and tumor characteristics are listed in Table 1. Fifty-eight patients (median age 64.5, IQR 51-71 years) underwent conventional TURBT and 75 patients underwent Tm-fiber ERBT, (4 patients $(5.6 \%)$ were excluded due to conversion to TURBT (tumor posterior location)). Therefore, in the final analysis we have included 71 patients (median age 62.0, 53-68 years) after ERBT. There was no difference in age, tumor location distribution (Fig. 3), sex distribution, tumor stage and grade between the groups. Tumors were smaller in the conventional TURBT group compared to those in the Tm-fiber ERBT group (median 1.5, IQR 1.0-2.2 cm versus 2.0, IQR $1.5-3.0 \mathrm{~cm}, p=0.024)$. The number of tumors were comparable between the groups (median 1.0, IQR 1-3 and median 1.0, IQR 1-2, $p=0.23$ ). Twentyone of $71(29.6 \%)$ patients treated with conventional TURBT had multifocal bladder cancer compared to 14 of $58(24 \%)$ patients in the Tm-fiber ERBT group $(p=0.31)$. Mean ERBT duration was longer than TURBT -39.0 and $34.0 \mathrm{~min}$, respectively $(p=0.045)$.

Median follow up was 12 months (IQR, 3-24). Patients treated with Tm-fiber ERBT had better RFS rates than those treated with conventional TURBT at 3 months $(97.2 \%$ and $84.5 \%, p=0.011)$ and 6 months (91.5\% and 67.2\%, $p<0.001$ ) (Fig. 4).

Pathological examination revealed the presence of muscularis propria in $58.6 \%$ of cases after conventional TURBT compared to $91.5 \%$ of cases after Tm-fiber ERBT. Absence of the detrusor in the specimen always warranted re-TURBT. Univariable and multivariable analyses showed no association

Table 1

Patient characteristics in the TURBT and Tm-fiber ERBT groups. The values given represent $\mathrm{n}(\%)$ or mean \pm standard deviation (range) and for nonparametric values - median, IQR

\begin{tabular}{lccc}
\hline Parameter & TURBT $(n=58)$ & Tm-fiber ERBT $(n=71)$ & $p$ \\
\hline Males/ Females (\%) & $17.2 / 82.8$ & $26.8 / 73.2$ & 0.141 \\
Age, median, IQR (years) & $64.5(51-71)$ & $62.0(53-68)$ & 0.415 \\
Tumor size, median, IQR (cm) & $1.5(1.0-2.2)$ & $2.0(1.5-3.0)$ & $0.024^{*}$ \\
Number of tumors, median, IQR & $1.0(1-3)$ & $1.0(1-2)$ & 0.226 \\
Surgery duration, mean, SD & $34.0( \pm 8.8)$ & $39.0( \pm 16.5)$ & $0.045^{*}$ \\
Stage & & & \\
$\quad$ pTa & $30(51.7)$ & $39(54.9)$ & 0.426 \\
pT1 & $28(48.3)$ & $32(45.1)$ & \\
Tumor grade (1973)+ & $28(48.3)$ & $42(59.2)$ & 0.108 \\
$\quad$ G1 & $20(34.5)$ & $23(32.4)$ & \\
G2 & $10(17.2)$ & $6(8.5)$ & 0.089 \\
$\quad$ G3 & & & \\
Tumor grade (2004)+ & $36(62.1)$ & $52(73.2)$ & $18(25.4)$ \\
$\quad$ Low Grade & $22(37.9)$ & & \\
High Grade &
\end{tabular}

*-statistically significant difference. ${ }^{+}$WHO classification, 1973-2004. 

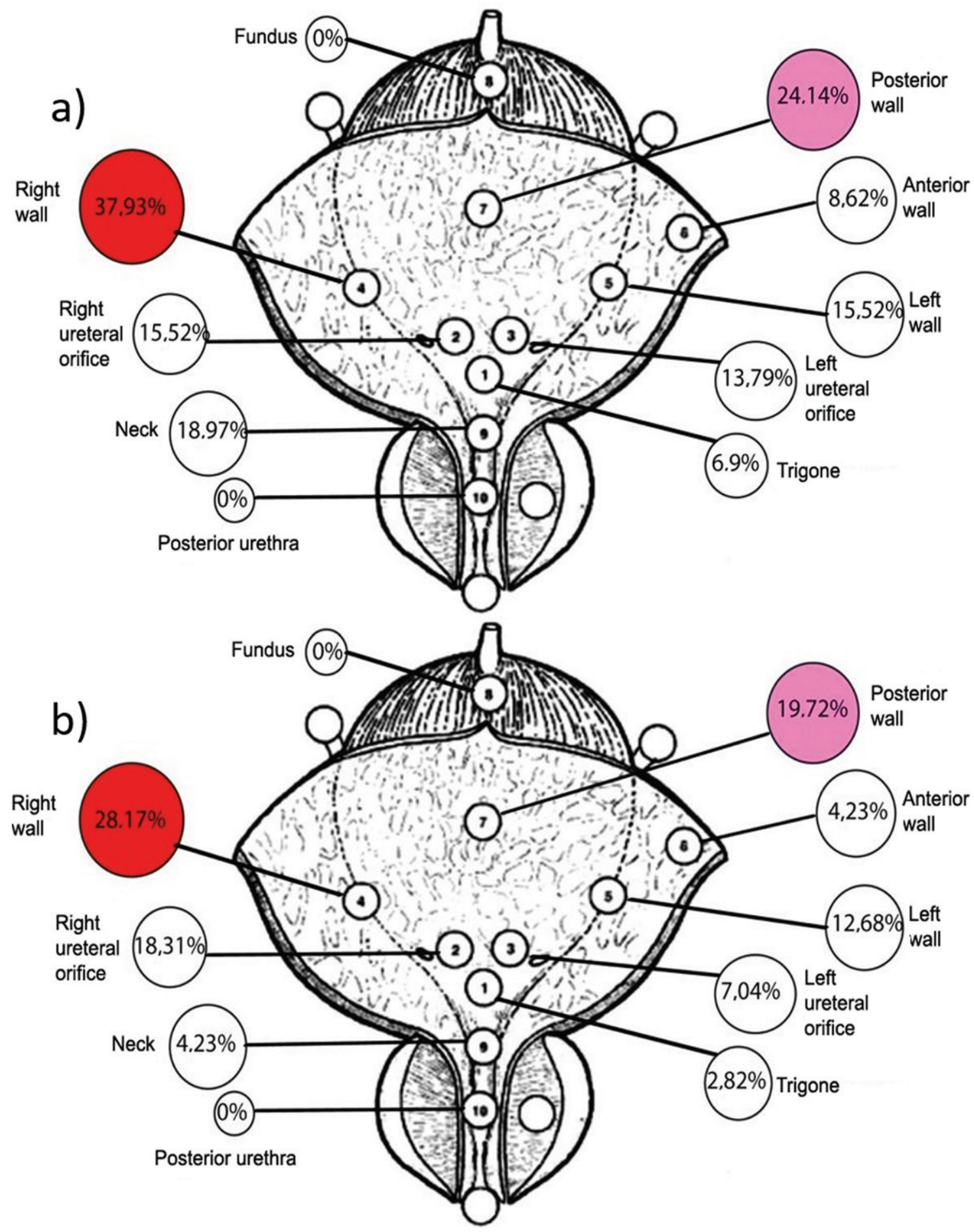

Fig. 3. Tumor location in the a) TURBT group; b) Tm-fiber ERBT group.

of RFS with sex, mitomycin C instillation, tumor location, concomitant CIS on pathology, patient age, tumor stage or grade. Conversely, the type of surgery
(HR 5.35; CI 95\% 1.47-19.44, $p=0.011$ ) and presence of muscular layer on pathology (HR 2.66; CI $95 \% 1.01-6.95, p=0.047$ ) was associated with RFS 


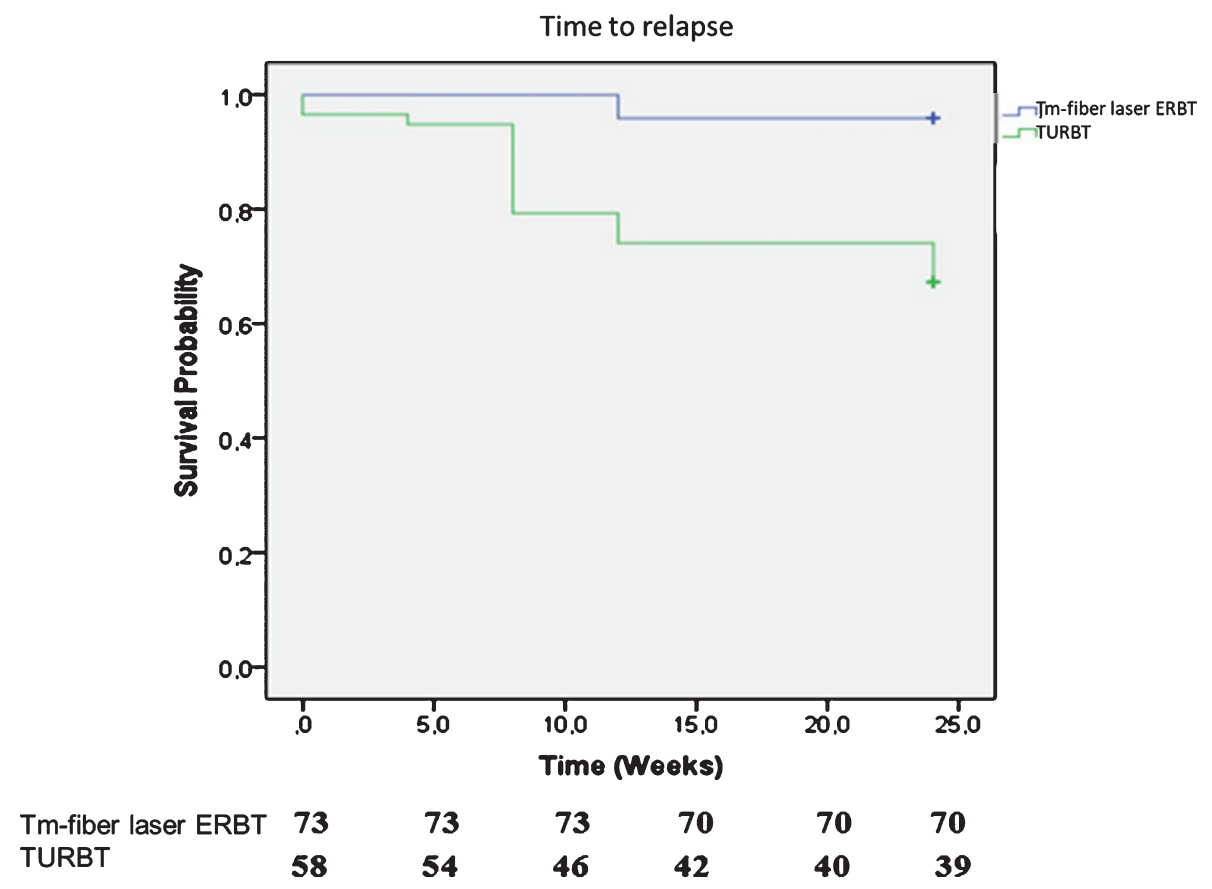

Fig. 4. Kaplan-Meier analysis for relapse-free survival.

Table 2

Univariable and multivariable analysis of recurrence

\begin{tabular}{|c|c|c|c|c|c|c|c|c|}
\hline & \multirow[t]{2}{*}{ No recurrence at $6 \mathrm{mo}$} & \multirow[t]{2}{*}{ Recurrence at $6 \mathrm{mo}$} & \multicolumn{3}{|c|}{ Univariable } & \multicolumn{3}{|c|}{ Multivariable } \\
\hline & & & $P$ & HR & CI $95 \%$ & $P$ & HR & CI $95 \%$ \\
\hline \multicolumn{3}{|c|}{ Surgical technique } & 0.001 & 9.104 & $2.692-30.789$ & 0.011 & 5.354 & $\overline{1.475-19.443}$ \\
\hline TURBT & 39 & 19 & & & & & & \\
\hline Tm-fiber ERBT & 65 & 6 & & & & & & \\
\hline \multicolumn{3}{|c|}{ Muscular layer on pathology } & 0.006 & 3.746 & $1.463-9.592$ & 0.047 & 2.656 & $1.105-6.952$ \\
\hline Found & 89 & 18 & & & & & & \\
\hline Not found & 11 & 7 & & & & & & \\
\hline \multicolumn{3}{|c|}{ CIS } & 0.430 & 1.796 & $0.420-7.685$ & 0.483 & 0.567 & $0.117-2.761$ \\
\hline Present & 5 & 2 & & & & & & \\
\hline Not present & 95 & 23 & & & & & & \\
\hline \multicolumn{3}{|c|}{ Staging } & 0.284 & 1.525 & $0.705-3.927$ & 0.113 & 1.932 & $0.855-4.362$ \\
\hline $\mathrm{Ta}$ & 47 & 11 & & & & & & \\
\hline $\mathrm{T} 1$ & 57 & 14 & & & & & & \\
\hline \multicolumn{3}{|c|}{ Tumor grade (2004) } & 0.099 & 1.552 & $0.921-2.616$ & 0.215 & 1.403 & $0.822-2.935$ \\
\hline Low grade & 48 & 10 & & & & & & \\
\hline High grade & 56 & 15 & & & & & & \\
\hline
\end{tabular}

(Table 2). No differences between the relapse rates in patients with large sized tumors were observed.

Peri- and postoperative parameters are shown in Table 3. The catheter was usually removed at 24 hours after surgery. The obturator nerve reflex was observed in 10 patients from the conventional
TURBT group and no patients in the Tm-fiber ERBT group.

In the conventional TURBT group, $6(10.3 \%)$ patients showed radiographic evidence (leakage on cystography which was only done in cases of intraoperative bladder injury) of perforation but none had 
Table 3

Peri- and postoperative parameters in the TURBT and Tm-fiber ERBT groups

\begin{tabular}{lcccccc}
\hline & \multicolumn{2}{c}{ TURBT $(n=58)$} & & Tm-fiber ERBT $(n=71)$ & \multirow{2}{*}{$p$} \\
\cline { 2 - 3 } & $\mathrm{N}$ & $\%$ & & $\mathrm{~N}$ & $\%$ & \\
\hline Obturator nerve reflex & 10 & 17.2 & & 0 & 0.00 & $<0.001^{*}$ \\
Perforation & 6 & 10.3 & & 0 & 0.00 & $0.007^{*}$ \\
Acute bleeding & 3 & 5.2 & & 0 & 0 & 0.088 \\
UTI & 5 & 8.6 & & 6 & 8.4 & 0.607 \\
Detrusor presence & 34 & 58.6 & & 65 & 91.5 & $<0.001^{*}$ \\
Catheterization time, median (hours) & 24 & & & 24 & & 0.312 \\
Hospital stay, median (days) & 4 & & & 4 & & 0.210 \\
*-statistically significant difference. & & & & &
\end{tabular}

any clinical signs/symptoms. Three $(5.2 \%)$ patients developed bleeding that required coagulation with electrical loop. In these patients, no intravesical chemotherapy was administered. In the Tm-fiber ERBT group, there was no evidence of the obturator nerve reflex, radiographic perforation or bleeding that needed intervention. Urinary tract infections (UTI) were observed in both groups: in $8.6 \%$ of cases in the conventional TURBT group and in $8.4 \%$ of cases in the Tm-fiber ERBT group $(p=0.61)$.

\section{DISCUSSION}

Conventional TURBT is the current standard of care for NMIBC [9]. Despite its broad and longstanding use, it suffers from important limitations such as its neglect of the general principles of oncologic surgery. The fragmented, multistep resection of the tumor results in poor specimen quality (i.e., lack of integrity) while scattering free-floating tumor cells in the bladder, which are thought to cause secondary implantation and relapse [10]. To overcome these limitations, the concept of complete tumor removal with EBRT was developed. This technique has been postulated to decrease the dispersion of tumor cells, thereby lowering the risk of disease recurrence and progression [6]. In contrast to electrical surgical techniques, laser surgery is characterized by less damage to the specimen because of its significantly lower penetration depth. To assess the potential benefits of Tm-fiber ERBT as a treatment option in papillary NMIBC, we performed a prospective non-randomized phase II study in a consecutive cohort of patients treated for primary $\mathrm{Ta}$ or $\mathrm{T} 1$ NMIBC.

In agreement with previous studies, we found that Tm-fiber ERBT resulted in significantly lower rates of disease recurrence at 3 and 6 months after surgery compared to conventional TURBT $[11,12]$. This benefit remained true even after adjusting for the effects of standard clinicopathologic features including tumor stage, grade, and concomitant CIS, among others. We detected a rate of residual tumor after conventional TURBT that was within the range reported in the literature [13]. Bladder cancer recurrence has been shown to range between 50 and $80 \%$, with residual tumors on re-resection (performed at 2 to 6 weeks after the initial resection) ranging between 33 and $53 \%$ for T1 HG tumors and up to $40 \%$ for Ta HG tumors [14-16]. The lower rate of recurrence with Tm-fiber ERBT could be due to undetected biases as the sample size is small and the study was not randomized. However, it is also likely that the benefit is real and therefore needs confirmation in larger prospective randomized multicenter phase III trials. Therefore, based on these promising results, we started enrollment in such a trial (En-bloc vs Conventional Resection of Primary Bladder Tumor (eBLOC); ClinicalTrials.gov identifier: NCT03718754).

We found that Tm-fiber ERBT does less thermal damage and preserves tissue integrity more commonly than conventional TURBT. Indeed, a wellknown disadvantage of conventional TURBT is its severe thermal damage to cellular and extracellular structures. The TURBT tissue submitted to the pathologists is often of poor quality making interpretation of the results difficult to impossible. Key features that would be necessary to collect for adequate risk assessment and therapy planning such as lymphovascular invasion and variant histology are often not interpretable [17-20]. One of the essential features for clinical decision-making is the invasion status of the lamina propria. All too often, due to inadequate specimen acquisition and/or the cautery artifact, it becomes impossible for the pathologist to ascertain tumor stage and the likelihood of having removed all the cancer [21]. Indeed, detrusor muscle cells in the specimen has become one of the main indicators of quality of NMIBC surgery 
$[11,22]$. According to the current data, muscularis propria is present in $50-86 \%$ of cases after conventional TURBT [9]. Not only does this put the patient at risk for understaging, but it also entails a repeated surgery to ensure all tumor removal prior to intravesical therapy [23]. Witjes et al. reported that patients treated with ERBT had detrusor in their specimen in over $90 \%$ of cases [23]. In our study, there was detrusor muscle on pathology in $58 \%$ of conventional TURBT cases compared to $91 \%$ of Tm-fiber ERBT cases $(p<0.001)$. This fact could lead to a lower rate of relapse at the resection site thereby improving prognosis.

We confirmed, in agreement with previous studies, that the absence of detrusor on pathology is independently associated with tumor relapse [16]. Analyzing the data from 2,410 patients from seven EORTC phase III trials, Brausi et al. suggested that the quality of the TUR performed by the individual surgeons may be responsible for the high variability in recurrence rates after TUR (persistence of tumor) [24]. ERBT could allow for not only better risk stratification because of better specimen quality but also a lower recurrence rate because of its more complete removal of the tumor. Thus, ERBT could help lower the need for a second-look resection as the entirety of the tumor is likely to have been removed at the first resection. Indeed, we found that residual tumors were detected in seven patients after TURBT and in one patient after Tm-fiber ERBT. Thus, ERBT may allow avoiding an unnecessary re-TURBT and needs to be tested.

The Tm-fiber laser used in ERBT has a low penetration depth due to its wavelength of $1940 \mathrm{~nm}$, which matches the peak of water absorption. It allows for minimal incision which is crucial during ERBT. Not only does this offer increased safety, but it is also associated with little to no risk of thermal damage to the specimen. Indeed, we found that carbonization and coagulation zones were minimal after Tm-fiber ERBT while specimens after standard transurethral electrosurgery had significant zones of thermal damage. This supports the statement that EBRT results in higher specimen quality for the pathologists who can now even determine margin status while maintaining patient safety.

We found that $17.2 \%$ of patients in the TURBT group had the obturator reflex. This is in accordance with the published data reporting a rate of $12-25 \%$ in patients undergoing bladder electrosurgery with a perforation rate of 2-10\% [25-27]. We also found a radiographic perforation rate of $10 \%$ with TURBT in contrast to a lack of obturator reflex and therefore no perforation with Tm-fiber ERBT.

The main limitations of the study were the comparatively small number of participants and lack of randomization. Another limitation was the absence of bipolar surgery. However, bipolar TURBT has not been shown to be safer than monopolar TURBT [28]. Also, neither photodynamic diagnosis (PDD), nor narrow-band imaging (NBI) were used. However, while they could have lowered the recurrence rates, it would have affected both groups equally.

\section{CONCLUSIONS}

Tm-fiber ERBT is a safe and effective therapeutic option for NMIBC. It is associated with a lower intraoperative complication rate, lower recurrence rate and better specimen quality compared to conventional TURBT. Moreover, it resulted in a higher rate of detrusor muscle presence, suggesting that it is a more "complete" intervention that may help avoid a re-resection that is currently the standard in all $\mathrm{T} 1$ HG and some Ta HG tumors. Tm-fiber ERBT may help overcome the current limitations of traditional TURBT resulting in more precise staging and a better prognosis for patients with NMIBC.

\section{ACKNOWLEDGMENTS}

The authors have no acknowledgments.

\section{FUNDING}

The authors report no funding.

\section{AUTHOR CONTRIBUTIONS}

DE: manuscript writting/editing, protocol development; MT: manuscript writting, protocol development, data analysis; VM: manuscript editing, protocol development; NS: data collection, protocol development, data analysis; LS: data analysis; NP: data collection, protocol development; DK: data collection, data analysis; VK: data collection, data analysis; LR: manuscript editing, protocol development; SFS: manuscript editing, protocol development; PG: protocol development.

\section{CONFLICT OF INTEREST}

The authors report no conflicts of interest. 


\section{REFERENCES}

[1] Antoni S, Ferlay J, Soerjomataram I, Znaor A, Jemal A, Bray F. Bladder Cancer Incidence and Mortality: A Global Overview and Recent Trends. Eur Urol. 2017;71(1):96-108.

[2] Ridge CA, Pua BB, Madoff DC. Epidemiology and staging of renal cell carcinoma. Semin Intervent Radiol. 2014;31(1):3-8.

[3] Tyson MD, Lee D, Clark P. New developments in the management of nonmuscle invasive bladder cancer. Curr Opin Oncol. 2017.

[4] Babjuk M, Burger M, Compérat E, et al. EAU Guidelines on Non-muscle-invasive Bladder Cancer (TaT1 and CIS) 2018. In: European Association of Urology Guidelines. 2018 Edition. Vol presented at the EAU Annual Congress Copenhagen 2018. Arnhem, The Netherlands: European Association of Urology Guidelines Office; 2018.

[5] Soria F, Pisano F, Gontero P, et al. Predictors of oncological outcomes in T1G3 patients treated with BCG who undergo radical cystectomy. World J Urol. 2018;36(11):1775-81.

[6] Kawada T, Ebihara K, Suzuki T, Imai K, Yamanaka H. A new technique for transurethral resection of bladder tumors: rotational tumor resection using a new arched electrode. J Urol. 1997;157(6):2225-6.

[7] Kramer MW, Rassweiler JJ, Klein J, et al. En bloc resection of urothelium carcinoma of the bladder (EBRUC): a European multicenter study to compare safety, efficacy, and outcome of laser and electrical en bloc transurethral resection of bladder tumor. World J Urol. 2015;33(12):1937-43.

[8] Fried NM, Murray KE. High-power thulium fiber laser ablation of urinary tissues at 1.94 microm. J Endourol. 2005;19(1):25-31.

[9] Babjuk M, Burger M, Zigeuner R, et al. EAU guidelines on non-muscle-invasive urothelial carcinoma of the bladder: update 2013. Eur Urol. 2013;64(4):639-53.

[10] Bryan RT, Collins SI, Daykin MC, et al. Mechanisms of recurrence of Ta/T1 bladder cancer. Ann R Coll Surg Engl. 2010;92(6):519-24.

[11] Soria F, Marra G, D'Andrea D, Gontero P, Shariat SF. The rational and benefits of the second look transurethral resection of the bladder for T1 high grade bladder cancer. Transl Androl Urol. 2019;8(1):46-53.

[12] Herrmann TR, Wolters M, Kramer MW. Transurethral en bloc resection of nonmuscle invasive bladder cancer: trend or hype. Curr Opin Urol. 2017;27(2):182-90.

[13] Xylinas E, Kent M, Kluth L, et al. Accuracy of the EORTC risk tables and of the CUETO scoring model to predict outcomes in non-muscle-invasive urothelial carcinoma of the bladder. Br J Cancer. 2013;109(6):1460-6.

[14] Grimm MO, Steinhoff C, Simon X, Spiegelhalder P, Ackermann R, Vogeli TA. Effect of routine repeat transurethral resection for superficial bladder cancer: a long-term observational study. J Urol. 2003;170(2 Pt 1):433-437.

[15] Kamat AM, Bagcioglu M, Huri E. What is new in nonmuscle-invasive bladder cancer in 2016? Turk J Urol. 2017;43(1):9-13.

[16] Rink M, Babjuk M, Catto JW, et al. Hexyl aminolevulinateguided fluorescence cystoscopy in the diagnosis and follow-up of patients with non-muscle-invasive bladder cancer: a critical review of the current literature. Eur Urol. 2013;64(4):624-38.

[17] Mari A, Kimura S, Foerster B, et al. A systematic review and meta-analysis of the impact of lymphovascular invasion in bladder cancer transurethral resection specimens. BJU Int. 2019;123(1):11-21.

[18] Mathieu R, Lucca I, Roupret M, Briganti A, Shariat SF. The prognostic role of lymphovascular invasion in urothelial carcinoma of the bladder. Nat Rev Urol. 2016;13(8): 471-9.

[19] Abufaraj M, Foerster B, Schernhammer E, et al. Micropapillary Urothelial Carcinoma of the Bladder: A Systematic Review and Meta-analysis of Disease Characteristics and Treatment Outcomes. Eur Urol. 2019;75(4):649-58.

[20] Abufaraj M, Shariat SF, Foerster B, et al. Accuracy and prognostic value of variant histology and lymphovascular invasion at transurethral resection of bladder. World J Urol. 2018;36(2):231-40.

[21] Hansel DE, Amin MB, Comperat E, et al. A contemporary update on pathology standards for bladder cancer: transurethral resection and radical cystectomy specimens. Eur Urol. 2013;63(2):321-32.

[22] Suarez-Ibarrola R, Soria F, Abufaraj M, et al. Surgical checklist impact on recurrence-free survival of patients with non-muscle-invasive bladder cancer undergoing transurethral resection of bladder tumour. BJU Int. 2019;123(4):646-50.

[23] Witjes JA, Bruins M, Compérat E, et al. EAU Guidelines on Muscle-invasive and metastatic Bladder Cancer 2018. In: European Association of Urology Guidelines. 2018 Edition. Vol presented at the EAU Annual Congress Copenhagen 2018. Arnhem, The Netherlands: European Association of Urology Guidelines Office; 2018.

[24] Brausi M, Collette L, Kurth K, et al. Variability in the recurrence rate at first follow-up cystoscopy after TUR in stage Ta T1 transitional cell carcinoma of the bladder: a combined analysis of seven EORTC studies. Eur Urol. 2002;41(5):523-31.

[25] Liu H, Wu J, Xue S, et al. Comparison of the safety and efficacy of conventional monopolar and 2-micron laser transurethral resection in the management of multiple nonmuscle-invasive bladder cancer. J Int Med Res. 2013;41(4):984-92.

[26] Chen X, Liao J, Chen L, et al. En bloc transurethral resection with 2-micron continuous-wave laser for primary nonmuscle-invasive bladder cancer: a randomized controlled trial. World J Urol. 2015;33(7):989-95.

[27] Chen J, Zhao Y, Wang S, et al. Green-light laser en bloc resection for primary non-muscle-invasive bladder tumor versus transurethral electroresection: A prospective, nonrandomized two-center trial with 36-month follow-up. Lasers Surg Med. 2016;48(9):859-65.

[28] Murugavaithianathan P, Devana SK, Mavuduru R, et al. Bipolar Transurethral Resection of Bladder Tumor Provides Better Tissue for Histopathology But Has No Superior Efficacy and Safety: A Randomized Controlled Trial. J Endourol. 2018;32(12):1125-30. 